\title{
Title: On the reproductive mechanism of Gram-negative protocells
}

\author{
Dheeraj Kanaparthi ${ }^{1 \& 2^{*}}$, Marko Lampe ${ }^{4}$, Baoli Zhu ${ }^{2 \& 4}$, Andreas Klingl ${ }^{5}$, Tillmann Lueders ${ }^{2 *}$ \\ \& Petra Schwille ${ }^{1^{*}}$
}

\begin{abstract}
Affiliations:
${ }^{1}$ Max-Planck Institute for Biochemistry, Munich, Germany.

${ }^{2}$ Chair of Ecological Microbiology, BayCeer, University of Bayreuth, Germany. Germany.

${ }^{4}$ Key Laboratory of Agro-ecological Processes in Subtropical Regions, CAS, Changsha, China.

${ }^{5}$ Department of Biology, LMU, Planegg-Martinsried, Germany.
\end{abstract}

${ }^{3}$ Advanced Light Microscopy Facility, European Molecular Biology Laboratory, Heidelberg,

\begin{abstract}
One of the major challenges of understanding early life or a bottom-up approach to creating synthetic life is to drive the process of reproduction that is entirely dependent on the chemical and physical properties of cell constituents. To achieve this objective, here we transformed cells into a sack of cytoplasm devoid of molecular biological mechanisms regulating their reproduction. Given such primitive cells could only have existed on early Earth, we grew then under Archean Eon environmental conditions. Results of our demonstrate that cells can reproduce very efficiently in the absence of molecular biological mechanisms. The method of their reproduction could be explained by chemical interactions between different cell constituents and by laws of physical chemistry, rather than the information encoded in their genome. Given the simplicity and efficiency of the process, we
\end{abstract}


propose that primitive cells inhabiting early Earth (protocells) could have reproduced by this process.

Introduction: Life on Earth is thought to have emerged in a series of steps described by the theory of chemical evolution ${ }^{1}$. According to this theory organic compounds required for building a cell were synthesized by Miller-Urey² like processes and subsequent prebiotic chemical reactions $^{3-5}$. Eventually these organic compounds coalesced to form a protocell ${ }^{6,7}$. These protocells were thought to have been very primitive and can essentially be described as lipid vesicles with internal chemical reactions. Unlike the present-day cells, whose morphology, growth, and reproductive processes are tightly controlled by molecular biological processes, such processes in protocells are thought to have been determined by physical and chemical properties of its constituents ${ }^{7,8}$. Despite being a key evolutionary link between prebiotic chemical reactions and prokaryotes, protocells to date remained a theoretical possibility with no definitive proof for their existence, nor was it demonstrated that physical and chemical properties of cell constituents alone could mediate complex biological processes like cell replication. In the present study we attempted to achieve cell fission into metabolically viable daughter cells mediated entirely by physicochemical properties of cell constituents.

Our study differs from earlier studies on protocells in two key aspects. First, rather than the conventional choice of using lipid vesicles, here we used protoplasts of the Gram-negative bacterium Rhodobacter sphaeroides $(R S-P)$ as a proxy protocell. In our opinion, bacterial protoplasts better simulate a primitive protocell than lipid vesicles for several reasons. Protoplasts are physiologically and chemically more complex than lipid vesicles. Most likely, even the most primitive self-replicating protocells should have been more complex in their 
chemical composition than typical lipid vesicles. Moreover, bacterial protoplasts like protocells are devoid of all molecular biological mechanisms either to regulate its shape or reproductive process ${ }^{9,10}$. As a second novelty of our study we grew these protoplasts under environmental conditions of early Earth, as environmental conditions are known to determine chemical properties of cell constituents ${ }^{11}$ and cells reproducing by these processes could only have been native to early Earth.

The exact environmental conditions of early Earth is a matter of debate among researchers; nevertheless, recent consensus among researchers suggests that surface temperatures of Archaean Earth (4000-2500 million years ago) ranged between $26^{\circ}$ to $35^{\circ} \mathrm{C}^{12,13}$ and Archaean Eon ocean were 1.5 to 2 times saltier than the current oceans ${ }^{14}$. To replicate these conditions, we grew $R S-P$ in half strength nutrient broth (NB) supplemented with 5-10\% Dead Sea salt (DSS) at $30^{\circ} \mathrm{C}$. DSS was used in our incubations rather than pure $\mathrm{NaCl}$ to replicate complex salt composition of Archaean oceans ${ }^{15,16}$. Ones we had obtained stable protoplasts $(R S-P)$, we transferred them into fresh growth media and monitored their morphological transformation at regular intervals. In the below sections of the manuscript, we present an in-depth morphological transformation of $R S-P$, followed by an explanation of the physical and chemical processes driving such transformations.

\section{Results and Discussion:}

Morphological transformation of $R S-P$ was monitored at regular intervals by staining observing the cells under a super-resolution STED microscope. Cells for STED microscopy were stained with membrane dye, $\mathrm{FM}^{\mathrm{TM}} 5-95$ and nucleic acid stain, PicoGreen ${ }^{\mathrm{TM}}$. A simplistic version of $R S-P$ 's life cycle is presented in Figure 1. 

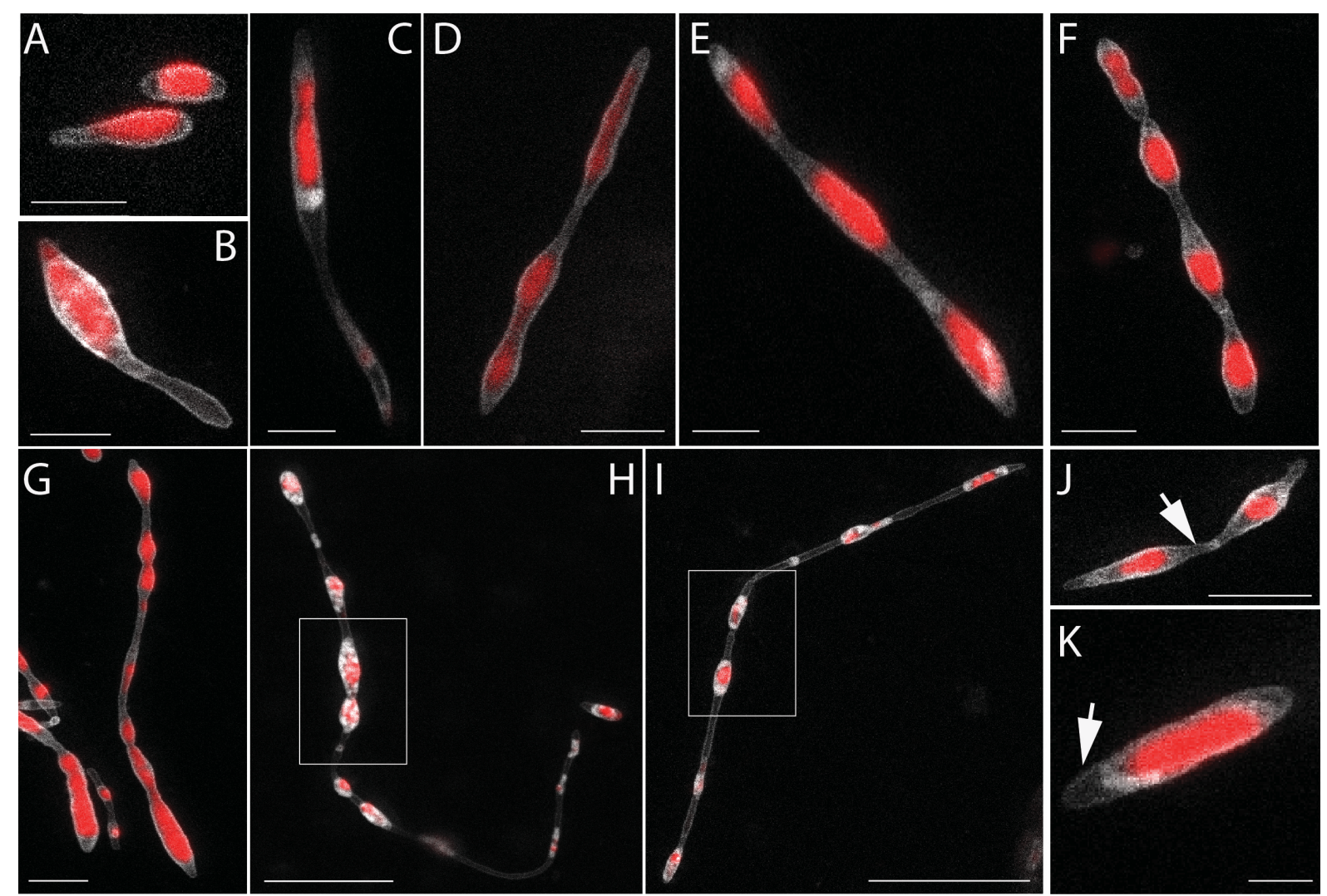

Figure 1. Life cycle of filamentous $R S-P$ cells. STED microscope Images (A-K) show life cycle of tubular and filamentous $R S-P$. Cells in these images were stained with universal membrane stain, FM $\mathrm{FM}^{\mathrm{TM}}$-95 (white) and DNA stain, PicoGreen ${ }^{\mathrm{TM}}$ (red). Arrows in images J points to collapsed hollow membrane, between the cytoplasmic compartments (daughter cells). Boxes in images H\&I show cytoplasmic compartments within the cell undergoing binary fission. Arrows in images K points to remnants of such excess membrane in daughter cells. Scale bars: $1 \mu \mathrm{m}(\mathrm{A}-\mathrm{G}, \mathrm{I} \& \mathrm{~J})$ and $10 \mu \mathrm{m}(\mathrm{H} \& \mathrm{I})$.

During the lag phase, growth was restricted to the outer membrane (OM) of the cell, which expanded to form a hollow filamentous extension (Figure 1B, C \& S1). The length of this tail varied from cell-to-cell, ranging between 0.5 to $5 \mu \mathrm{m}$ (Figure 1B, 1C \& Figure S1A). Based on our observations we propose a two-step process by which the hollow membrane extensions were formed. In the first step, disproportionately high lipid synthesis in comparison to cytoplasmic volume increase (low growth rate during lag-phase) had 
facilitated the transformation of excess membrane into vesicles ${ }^{17}$ extending either into or out of the cell (into the space between outer and inner membranes) (Figure S1G). As similar phenomenon of excessive lipid synthesis was reported among bacterial protoplasts ${ }^{18}$.

Subsequently hollow vesicles within the periplasmic space fused and incorporated into the outer membrane, leading to the formation of hollow filamentous extensions (Figure 1B \& Figure S1 E-G).
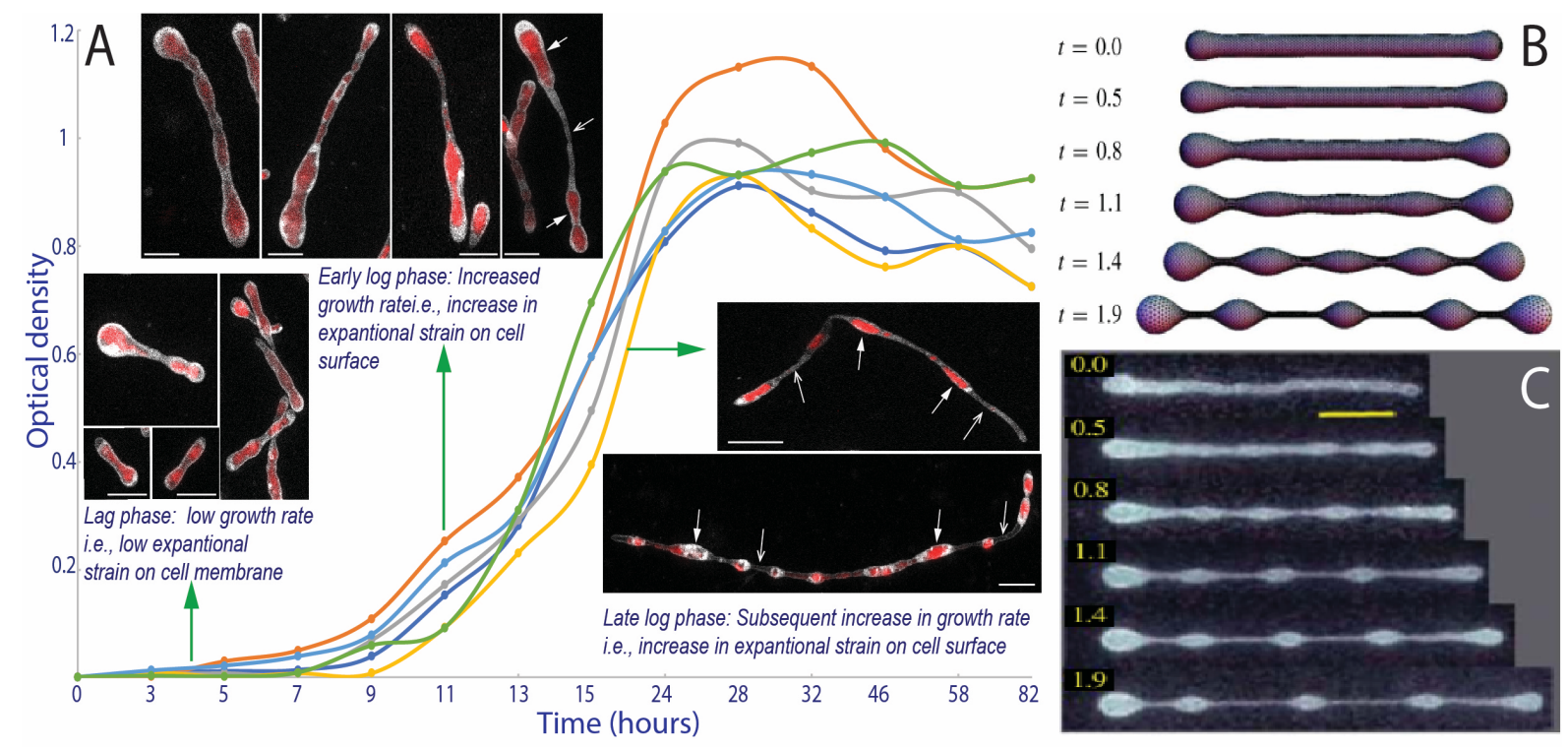

Figure 2. Image A show different growth stages of $R S-P$. Green arrows in the image points to the $R S-P$ morphologies observed during these growth stages. Closed and open arrows in the image points to regions of the cell with cytoplasm and hollow OM connecting the cytoplasmic compartments (also shown in Figure S2). Images B \& C show theoretically predicted, and experimentally observed morphologies of vesicles subjected to elongational stress (originally published by Narsimhan et al., $2015^{19}$ and Kantsler et al., 2008 20 ). Scale bar: lag-phase $(1 \mu \mathrm{m}), \log$-phase $(10 \mu \mathrm{m})$.

During the early-log phase, with an increase in the growth rate, volume of the cytoplasm increased, gradually filling in the hollow template created by the OM (Figure 1C, D \& Figure 
S1A, B, E \& G). This led to the sequential transformation of spherical cells in sequence into prolate or tubular cells and then into filamentous cells with cytoplasm mostly restricted to either ends of the cell, resembling dumbbells with long filamentous connections (Figure 2, early $\log$-phase).

In the subsequent growth stages, cells developed surface depressions along their length resembling tubular vesicles subjected to curvature-induced destabilization at their surface (like Rayleigh-Plateau instability) (Figure 1D-G \& 2-late log phase). These surface depressions were formed as a result of cytoplasmic compartment surrounded by inner membrane undergoing binary fission (Figure 1 D-I). In some filamentous cells we observed cytoplasmic compartments undergoing a symmetric division leading to the formation of daughter cells of equal sizes (Figure 1E \& F). In such cells, surface depressions are uniform in size and regularly spaced. Alternately, some cells developed unevenly sized depressions with a large lenticular structure either at the tip (Figure 3, S1B-D \& S3) or center of the filament (Figure 3\& S3). Such structures were formed due to asymmetric division of cytoplasmic compartments. We also observed some tubular cell undergoing stretching to form thin filamentous cells (Figure S4). Over time these surface depressions grew in depth and two adjacent cytoplasmic compartments were only linked to each other only by a hollow strand of outer membrane (boxed regions in Figure 1H \& Figure 2 - late log phase). rupture of outer membrane leading to the formation of individual daughter cells (Figure 1H-K).

The above-described morphological transformation observed in $R S-P$ can be explained either from the prospective of fluid mechanics ${ }^{19,20}$ or by Area differential elasticity (ADE) theory $^{21,22}$. Growth (or expansion) of cytoplasmic compartment gradually filling the void left 

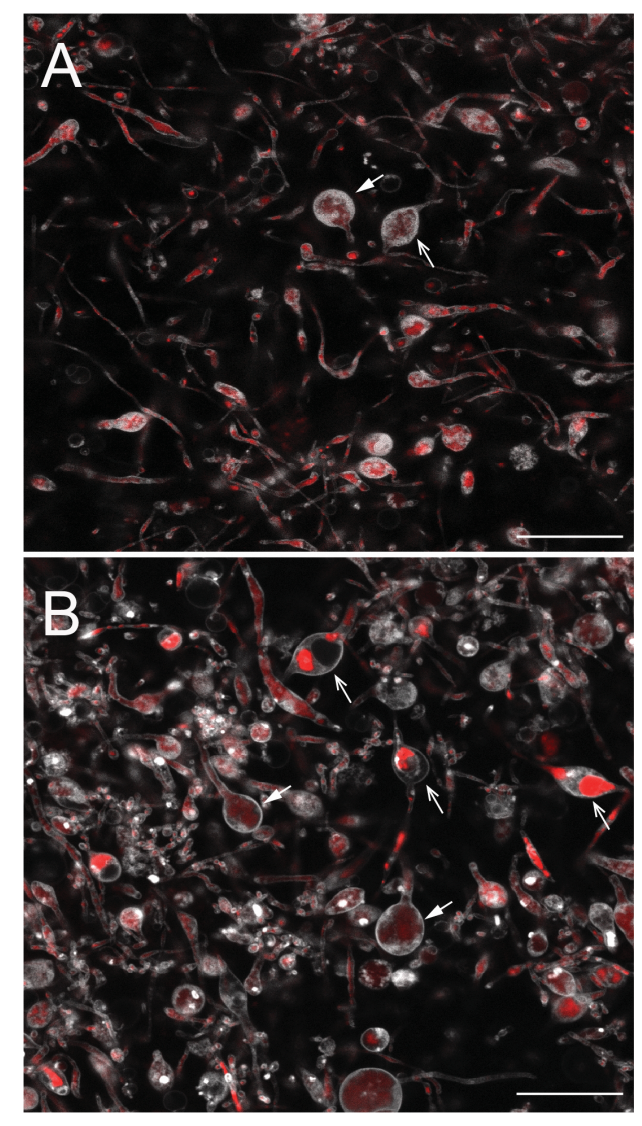

Figure 3. Images A\&B show RS-P cells in their early and late stationary growth stage.

Images show lenticular cells within the filamentous extensions either at one (closed arrows) or both ends (open arrows). Most lenticular cells contain intracellular membrane vesicles, giving them a reticulate appearance. Cells were stained, and color coded as described in Fig. 1. Scale bars: $10 \mu \mathrm{m}$.

by outer membrane resembles vesicle stretching under extensional flow (Figure 2, S2 \& S9). Morphological transformation of vesicles undergoing such stretching is dependent on expansional strain and elasticity of their membranes ${ }^{19,20}$. Vesicles experiencing a low elongational strain are shown to transformed into prolate or tubular shapes ${ }^{20}$. However, at a certain strain level (threshold strain), vesicles transformed into a dumbbell morphology. Any further increase in the strain, vesicles formed elongated dumbbells with beaded structures appearing along its length. Morphological transformation of $R S-P$ exhibited a similar pattern 
(Figure $2 \&$ S5). High and low elongational strains on the membrane translate into high and low growth rates. In accordance with our presumption, short prolate or tubular cells were most frequently observed during an early growth stage (lag-phase), when the growth rate of the cell was slower compared to other stages (Figure 2). With an increase in their growth rate during the early log-phase $R S-P$ transformed into a dumbbell shape (Figure 2). During the late log-phase they formed elongated dumbbells with beaded structures along its length (Figure 2). Subsequent reduction in the growth rate during stationary phase, transformed $R S$ $P$ into string of beads morphology (Figure $1 \mathrm{H} \& \mathrm{I}, 2)$. Based on these observations, we propose, growth rate as one of the factors regulating the morphology of $R S-P$.

The morphological transformation observed in $R S-P$ can also be explained by the Area differential elasticity (ADE) theory ${ }^{21,22}$. ADE theory states that when the area of the vesicle is smaller than that of the expected value, vesicles undergo shape deformation, leading to a series of morphological transformations. Amount of excess membrane area and intrinsic area difference (i.e., area difference between the expected and the actual area of the vesicle) determine the vesicle morphology. As predicted by ADE model ${ }^{21,22}$, spherical $R S-P$ cells transformed in sequence from prolate shape, into a cylindrical or a dumbbell shapes and finally into "string of beads" like morphologies (Figure 1\&2).

Staining $R S-P$ with dyes specific for fluid $\left(\mathrm{L}_{\mathrm{d}}\right)$ and universal membrane dyes showed that, this morphological transformation was accompanied by membrane phase-separation, which is also in accordance with ADE theory ${ }^{23}$ (Figure 4). During early growth stages, cell membrane underwent phase-separation with $\mathrm{L}_{\mathrm{d}}$ regions forming small patches within the cell membrane (Figure 4A). In the subsequent growth stages $\mathrm{L}_{\mathrm{d}}$ membrane coalesced around the cytoplasmic compartment and the outer membrane in these cells was largely composed of $\mathrm{L}_{0}$-membrane 
(Figure 4B-D). In cells of subsequent growth stages and cells undergoing binary fission, presence of $\mathrm{L}_{\mathrm{d}}$-membrane was restricted to the poles (Figure $4 \mathrm{E} \& \mathrm{~F}$ ), where membrane experiences a high spontaneous curvature and central barrel connecting the poles was noticed

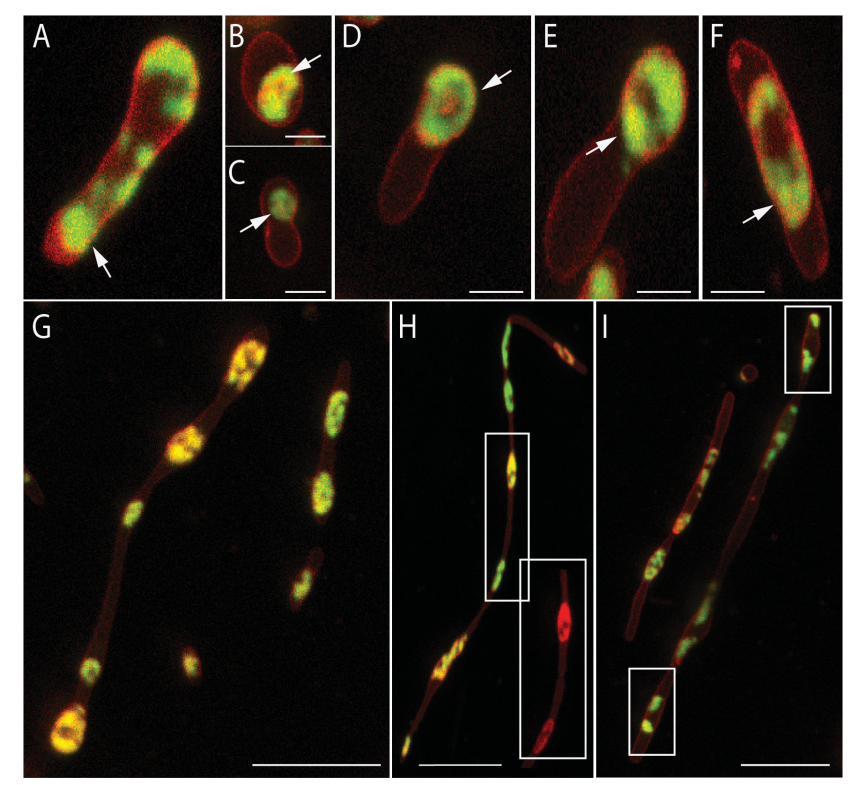

Figure 4. Images A-I show RS-P cells of different growth stages. Cells in these images were stained with universal membrane stain, $\mathrm{FM}^{\mathrm{TM}} 5-95$ (red) and membrane stain that specifically stains $\mathrm{L}_{\mathrm{d}}$ membrane, $F A S T^{\mathrm{TM}}$ Dil (green). Insert in image $\mathrm{H}$ show the region of the filament stained only with universal membrane stain. Boxed regions in image I show cytoplasmic compartments enclosed by inner cell membrane. $\mathrm{L}_{\mathrm{d}}$ membrane within these compartments is localized at the poles. Scale bars: $1 \mu \mathrm{m}(\mathrm{A})$ and $10 \mu \mathrm{m}(\mathrm{C}-\mathrm{D})$.

to be composed of $\mathrm{L}_{0}$-membrane (Figure $4 \mathrm{E} \& \mathrm{~F}$ ). Given the $\mathrm{L}_{\mathrm{d}}$ and $\mathrm{L}_{\mathrm{o}}$ membranes are largely composed of unsaturated and saturated phospholipids, we presume lipid molecules in the membrane underwent self-organization with unsaturated phospholipid rich $\mathrm{L}_{\mathrm{d}}$ membrane translocating to regions of the cells with high spontaneous curvature and distribution of $\mathrm{L}_{\mathrm{o}}$ membrane being restricted to linear regions of the cell membrane. Similarly, localization of $\mathrm{L}_{\mathrm{d}}$ and $\mathrm{L}_{\mathrm{o}}$ membranes selectively in inner and outer membrane of the cell could also be 
explained by a combination of physical properties of cytoplasm and organizational principles leading towards a stable and lowest free energy state. Given the constant movement of the cytoplasmic compartment of the cell (Movie S1 \& S2), it could have been energetically favorable to encapsulate cytoplasm with more fluid $\mathrm{L}_{\mathrm{d}}-\mathrm{membrane}$ rather than a rigid $\mathrm{L}_{\mathrm{o}^{-}}$ membrane.

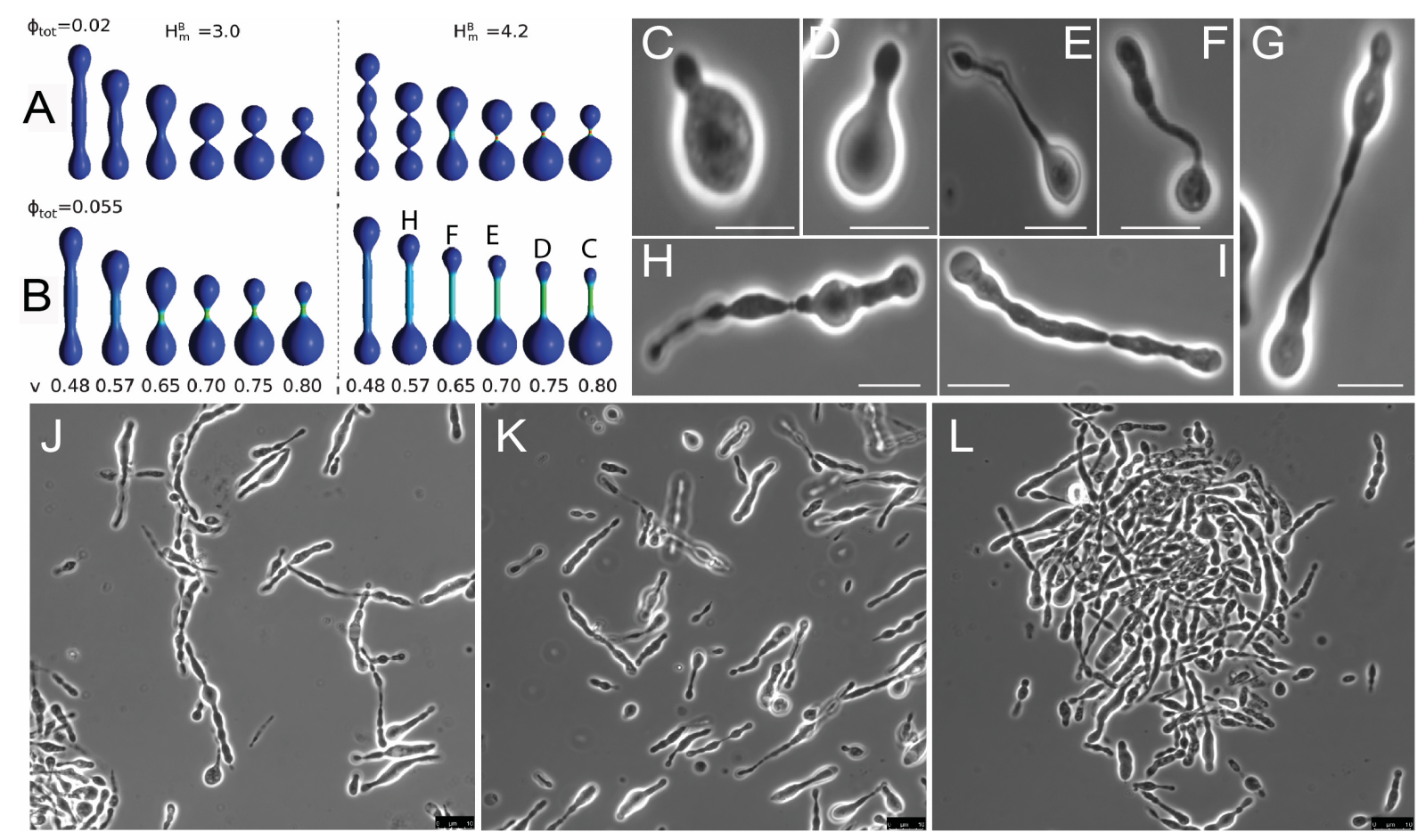

Figure 5. Images A\&B show morphologies of vesicles predicted by mathematical modelling ${ }^{24}$. The $\mathrm{V}$-values represent ratio of the volume of the vesicle to volume of the same vesicle in its spherical form, $\Phi_{\text {tot }}-$ represent the concentration of anisotropic component, $\mathrm{H}^{\mathrm{B}}{ }_{\mathrm{m}}$ - represent intrinsic curvature of the anisotropic component. Images C-G, show lag and early log phase cells, that were very similar to the mathematically predicted morphologies (indicated by figure C-H in image B). Decrease in the reduced volume (v) in live cells translated into gradual reduction of lipid: cell volume (from lag to log phase). Images J-K show early-log phase RS-P cells. Scale bars: $1 \mu \mathrm{m}(\mathrm{C} \& D)$ and $10 \mu \mathrm{m}(\mathrm{E}-\mathrm{L})$. 
Formation of large lenticular structures (Figure 3) could be explained by anisotropy in physical properties of cell membrane ${ }^{24}$ (Figure 5). Biophysical studies focused on understanding morphology and cell replication are mostly a result of studying LV's of rather simpler lipid composition. It was also generally assumed in such studies that the physical properties of LVs like fluidity and elasticity are isotropic along their surface. Unlike LV's, cell membranes have a complex lipid composition and contains numerous membrane associated proteins, which leads to lateral heterogeneity in their physical properties ${ }^{25}$. It was hypothesized by earlier studies that such differences could result in an un-uniform deformation of membrane surface leading to an asymmetric cell division and formation of lenticular structures ${ }^{24}$. In accordance with our proposition, morphological transformation of $R S-P$ is in tune with vesicle morphologies predicted by mathematical models of anisotropic membranes (Figure 5). High lipid to cytoplasmic volume of $R S-P$ during the early growth stages that represent a high value of V (reduced volume) and inherently high lateral heterogenicity $\left(\Phi_{\mathrm{tot}}\right)$ in membrane physical properties is expected for living cells due their complex lipid composition and by the presence of membrane proteins. A similar phenomenon of forming lenticular structures was reported earlier in urothelial cancer cells ${ }^{26}$.

These results suggest that organization of lipids within the cell membrane is determined by physiochemical properties of the lipid molecules rather than being dictated by molecular biological processes. Origin of distinctive phases and their coalescence into large rafts was proposed by earlier studies ${ }^{25}$. Despite observing such phases in LV's, existence of distinct phases in live cell membranes has been a point of contention among researchers ${ }^{27}$. To the best of our knowledge, ours is the first study to provide visual evidence of such distinct phases in membranes of live cells. Nevertheless, life cycle of $R S-P$, we presented above is consensual rather than literal. Over multiple reparations, we observed either complete omission of some 
of a growth stages or cells remained without transforming into another morphology for a prolonged period. Also, in contrast to our above argument that morphology of $R S-P$ is determined by its growth rate (by extension its growth stage), cell morphologies within any given growth stage were seldom uniform and in general are a mix of multiple morphologies (Figure 5J-L \& S6). Length and width of the cells differed considerable from one incubation to an another, even when cultivated under exact same media composition.

Morphological unpredictability of $R S-P$ could also be explained from a bio-physical prospective. Recent studies attempting to understand a similar phenomenon in LV's, suggested that the initial aspect ratio (width: height) of the vesicle determine its end morphology ${ }^{19,20}$. An ever-changing aspect ratio of the vesicles resulted in unpredictable end morphologies. During all growth stages, we observed $R S-P$ in a constant state of oscillation (ever changing aspect ratio) (Movie S1), which could also have contributed to the morphological diversity and unpredictability observed in our incubations.

Bacterial growth curves and their partition into different stages (lag, log \& stationary) in general represent growth dynamics of bacterial population. Nevertheless, averaging the data obscure considerable variation in the behavior of individual cells ${ }^{28,29}$. It is known that genetically identical cells within a single bacterial population could exhibit considerable cellto-cell variation in gene expression ${ }^{30,31}$ and their growth rates $^{32}$. Reason for such stochastic behavior of monoclonal cell has to date not been well understood. Manifestation of these variations in the morphology of normal bacteria could not be observed due to the presence of a rigid cell wall which confines cytoplasm of the cell into a pre-determined morphology. Given the absence of a cell wall in $R S-P$, we assume such cell-to-cell variations in growth rate is manifested in its morphology (Figure 5J-L \& S6), which could explain the morphological heterogenicity of cells within any given growth stage. 
To simulate Archaean Eon Ocean salinities, we grew $R S-P$ in $5-10 \%$ DSS-NB in all the above experiments. DSS is a mixture of several mono and divalent salts like $\mathrm{NaCl}, \mathrm{KCl}$ and $\mathrm{MgCl}_{2}$. These cations are known to interact differently with phospholipid membranes and are known to alter their physical properties ${ }^{11,33}$. Hence, in the subsequent experiments we attempted to understand if the morphological diversity exhibited by $R S-P$ during log and stationary growth phases was due to complex composition of DSS. To test this possibility, we repeated the above experiments replacing DSS with equivalent concentration of divalent and monovalent cationic salts like $\mathrm{MgCl}_{2}$ or $\mathrm{KCl} / \mathrm{NaCl}$. When grown in the presence of monovalent salts, $R S-P$ exhibited morphologies and reproduced by processes very similar to the ones observed when grown with DSS (Figure S7); except for forming thicker and longer filamentous cells with larger lenticular structures.

Similar cell morphologies were also observed when $R S-P$ was grown in $\mathrm{MgCl}_{2}$, with the exception that cell sizes were considerably smaller (Figure S8). Most cells observed in these incubations were of thin filamentous morphology. Thick tubular cells or lenticular cells with diameter $>1-2 \mu \mathrm{m}$ were rarely observed (sometimes only transiently during the mid-growth stages) (Figure S8). Unlike when grown in DSS or $\mathrm{KCl}$, cells in their last growth stage were mostly uniform in their morphology (Figure S8). Cells of this stage are predominantly unicellular daughter cells or cells in dyad undergoing binary fission. When $R S-P$ was transferred between growth media containing different salt compositions, morphologies of $R S-P$ changed reversibly. This suggests that salt composition of the media determined the morphology of cells rather than the molecular biological processes encoded in its genome. 
Different morphologies exhibited by $R S-P$ when grown in the $\mathrm{KCl}$ and $\mathrm{MgCl}_{2}$ could be due to the nature of interaction between phospholipid membranes and these cations ${ }^{11}$. It was reported that divalent cations like $\mathrm{Mg}^{+2}$ organizes lipids into clusters of 10-15 molecules $e^{e a c h}{ }^{34}$. This in turn dehydrates and increases the stiffness of the membrane making its area expansion energetically unfavorable. On the other hand, monovalent cations like $\mathrm{K}^{+}$are known to reduce bending rigidity of the membrane, making the membrane more flexible ${ }^{33}$. This phenomenon could explain easy expansion of cell membrane, conducive for formation of larger cells in the presence of $\mathrm{KCl}$ and smaller cell sizes in when grown in the presence of $\mathrm{MgCl}_{2}$.

Cells in all the experiments above were grown under static conditions. Given such conditions are rarely encountered in natural environments, we repeated the above experiments under multiaxial elongational flow conditions by growing $R S-P$ on an orbital shaker. Under these growth conditions, cells exhibited a more uniform morphology irrespective of the nature of the salt (Figure S9). Little morphological variation was observed during different growth stages. Most cells during lag, log and stationary growth stages were either individual cells or cells in dyad undergoing binary fission. Other cell morphologies were rarely observed.

Earlier studies proposed that fission of a protocells into daughter cells should have been achieved through physical forces. Hence to achieve protocell fission, these studies employed methods like extrusion through a narrow pore size filter or by application of external force 7,35,36. As most of the cells in the last growth stage are primarily unicellular daughter cells that were formed by fission of filamentous $R S-P$ and given the fact that our experiments were conducted under static conditions, we do not assume fission of cells was a result of an external stimulus. We rather propose that fission of daughter cells was achieved by forces 
internal to the cell and was a result of internal metabolic reactions ${ }^{37}$ or Brownian motion of intracellular constituents ${ }^{38}$. It was also hypothesized that presence of internal metabolism could lead to shape instabilities on the cell surface ultimately leading to cell division ${ }^{37}$. Morphologies and reproductive processes similar to the ones observed in $R S$ - $P$ were recently reported among lipid vesicles encapsulating nanoparticles, to simulate Brownian motion of proteins within a cell ${ }^{38}$. Uncanny morphological similarities between morphologies and reproductive processes of $R S-P$ with LV's encapsulating nanoparticles from above mentioned study, suggests that morphologies and reproductive processes could have been determined by the physical forces exerted by intracellular constituents. In support of this argument, we observed that cells or intracellular constituents were seldom found in a static state (Movie S1 $\& \mathrm{~S} 2)$.

Another factor that could have contributed to the fission of cells is the asymmetric conditions on either side of the cell membrane - organic carbon within the cell and inorganic salts outside the cell. Together with the movement of intracellular constituents, such conditions are known to induce constant deformation on the membrane surface ${ }^{33}$. In support of this presumption, addition of bactericidal compounds like sodium azide $(0.3 \%)^{39}$ or formaldehyde ${ }^{40}$ did not have any influence on the oscillation of the cells. These oscillations could only be halted when conditions inside and outside the cells were equilibrated by creating pores within the membrane using detergents like Triton X-100 (0.5\%), suggesting that asymmetric conditions on either side of the cell membrane contributed to the deformation of cells and provided the force required to mediate cell fission. 

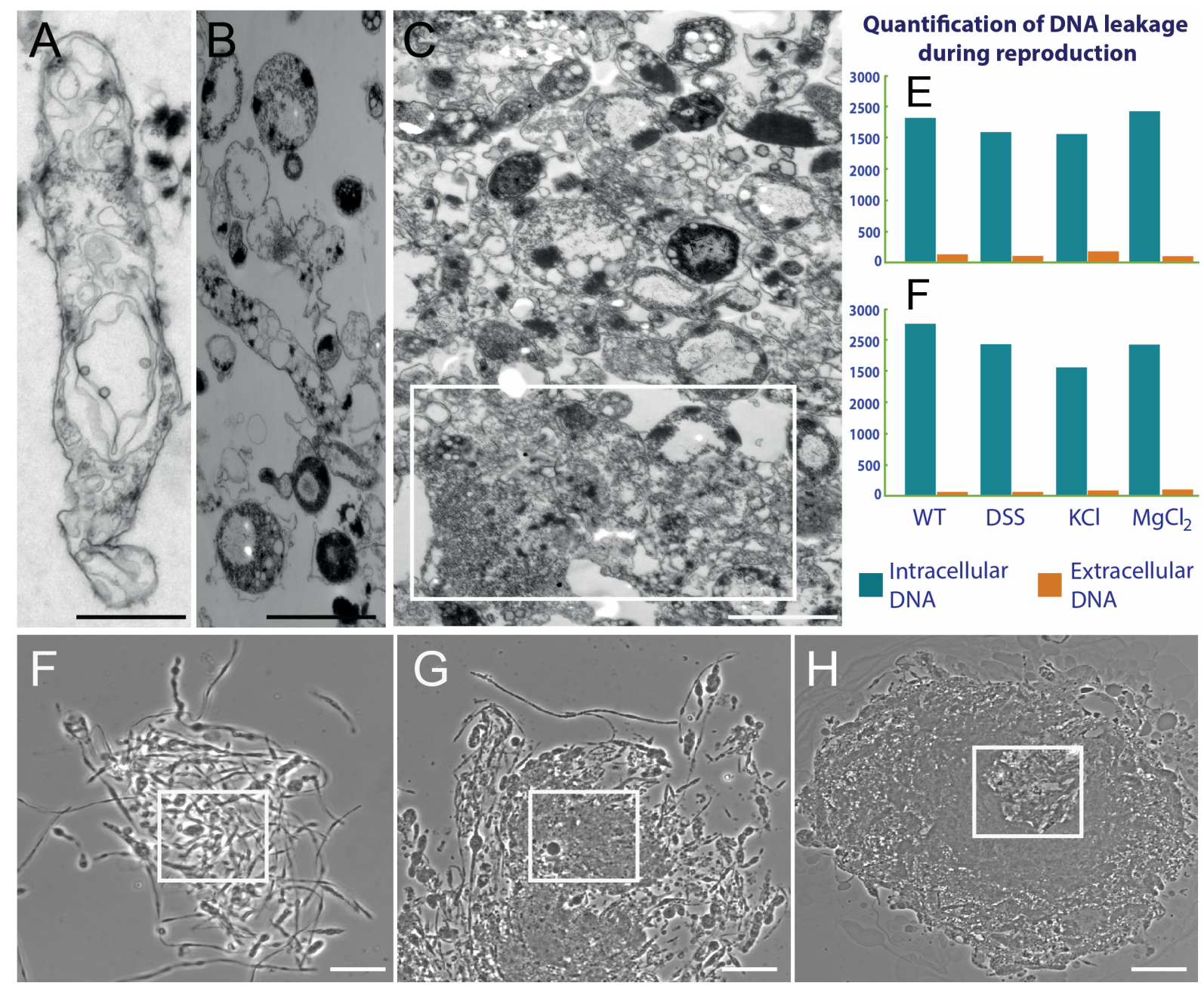

Figure 6. Images A-C are TEM images of $R S-P$ during their last growth stage. This stage consists of cells that were depleted in their cytoplasm and considerable amount of membrane debris (boxed region in C). Images F-G show sequence of steps involved in the formation of membrane debris. Images E\&F show averaged amount $(n=5)$ of extracellular and intracellular DNA, when $R S-P$ was grown under static conditions and while shaking (WT- $R$. sphaeroides grown in NB; DSS, $\mathrm{KCl} \& \mathrm{MgCl}_{2}-R S-P$ grown in the presence of respective salts). Scale bar: $1 \mu \mathrm{m}(\mathrm{A}-\mathrm{C}) \& 10 \mu \mathrm{m}(\mathrm{F}-\mathrm{H})$.

Based on the above inferences we argue that fission of parent cell into daughter cells was mediated by physiochemical forces generated by virtue of processing internal metabolism ${ }^{37}$, 
random movement of cytoplasmic constituents (Movie S2), deformations caused by transient binding of cations to the membrane surface ${ }^{41}$ and asymmetric environmental conditions on either side of the cell membrane in terms of osmolarity or nature of the solute ${ }^{33}$. All these conditions are shown to generate shape instabilities, which could have led to fission of cells ${ }^{37}$ (Movies S3). Nevertheless, relative contribution of each of these processes to the fission of parent cells is hard to determine in live cells, especially given their metabolic and compositional complexity.

Reproductive efficiency of $R S-P$ is determined by quantifying the leakage of cell constituents during the process ${ }^{7}$. Comparison of DNA concentrations within and out of the cell during late $\log$ phase (Figure $6 \mathrm{E} \& \mathrm{~F}$ ), suggests that $R S$ - $P$ reproduced with a similar efficiency as cell in their native form with functioning molecular biological mechanisms. Given the considerable amount of membrane debris (Figure 6) observed during the stationary growth phase, we propose that $R S-P$ reproduced rather inefficiently. Nevertheless, reproductive efficiency was dependent on the environmental conditions (Figure 6, S8 \& S9). Relatively less wastage of cell constituents and production of membrane debris was observed when $R S$ - $P$ was grown in the presence of $\mathrm{MgCl}_{2}$ (Figure S8) than when cultured in DSS or $\mathrm{KCl}$. Reproductive efficiency was also higher when $R S-P$ was cultured under non-static conditions (Figure S9). Despite the wastefulness of its reproduction process under some environmental conditions, all our incubations resulted in viable daughter cells with cytoplasmic activity and intracellular DNA (Figure 1, 2, 4, \& 7). Subsequent transfer of these cells either directly or after passing through $0.44 \mu \mathrm{m}$ filter (Figure 7) to separate daughter cells from other large cells and membrane debris into fresh media always resulted in growth. This mechanism of reproduction and the efficiency of the process is comparable to theoretical propositions of protocell reproduction ${ }^{7}$, proposed from studying LV's. 

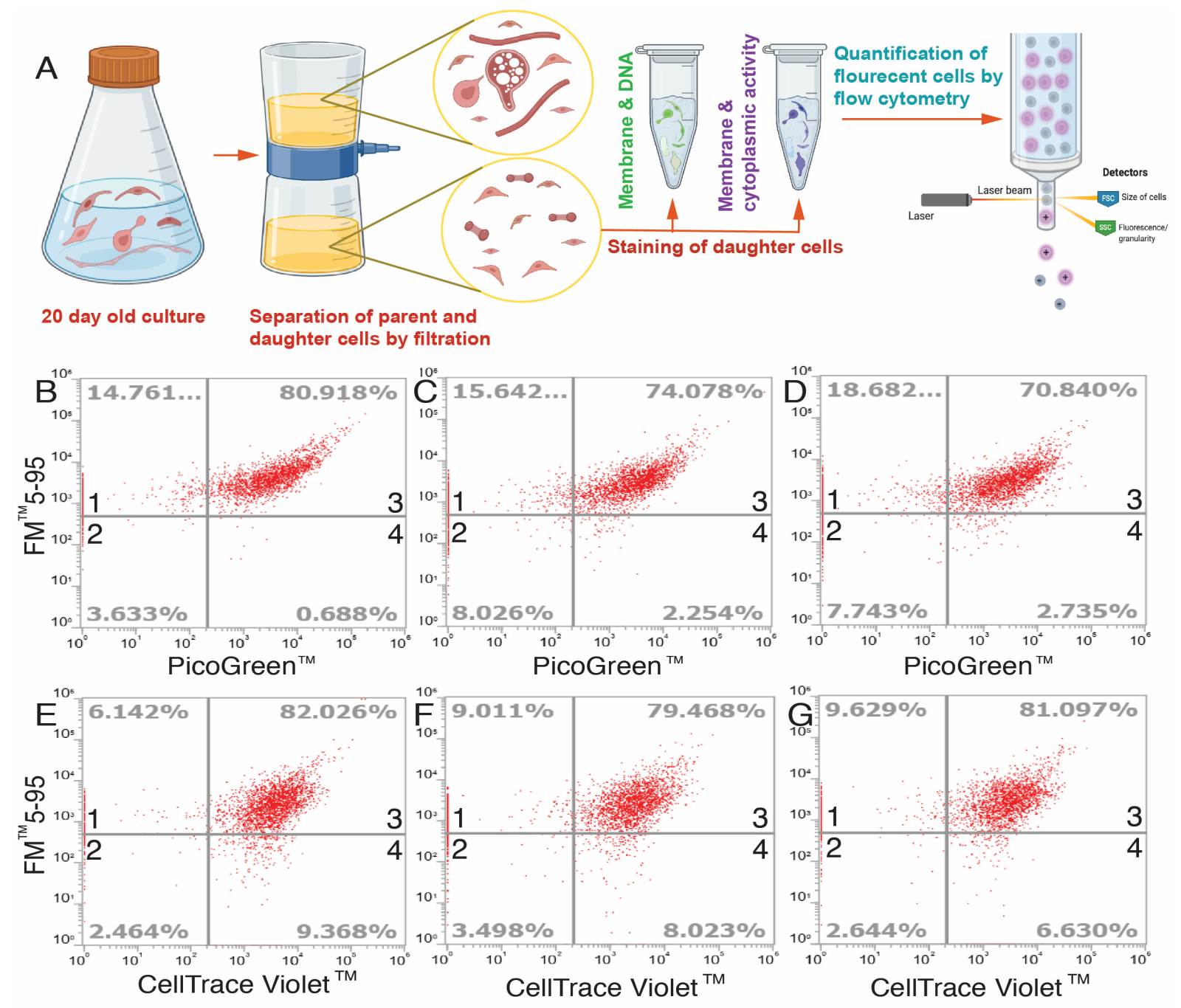

Figure 7. Panel A show the workflow of the experiment. Images b-g show flowcytometry plots. Quadrants 1, 2, $3 \& 4$ in each plot represent the percentage of cells stained with only FM $^{\mathrm{TM}} 5-95$ (1), cells stained only with PicoGreen ${ }^{\mathrm{TM}}$ (2), cells stained by both $\mathrm{FM}^{\mathrm{TM}}$ 5-95 \& PicoGreen $^{\mathrm{TM}}$ (3) and cells that weren't stained either by $\mathrm{FM}^{\mathrm{TM}} 5-95$ \& PicoGreen $^{\mathrm{TM}}$ (4). Images d-f show flowcytometry plots, quadrants $1,2,3 \& 4$ in each plot represent the percentage of cells stained with only $\mathrm{FM}^{\mathrm{TM}} 5-95$ (1), cells stained with only CellTrace ${ }^{\mathrm{TM}}$ Violet (2), cells stained by both $\mathrm{FM}^{\mathrm{TM}} 5-95$ \& CellTrace ${ }^{\mathrm{TM}}$ Violet (3) and cells that were not stained either by $\mathrm{FM}^{\mathrm{TM}} 5-95$ \& CellTrace ${ }^{\mathrm{TM}}$ Violet (4). Image G show RS-P daughter cells stained with $\mathrm{FM}^{\mathrm{TM}} 5-95$ \& PicoGreen ${ }^{\mathrm{TM}}$ after passing through $0.44 \mu \mathrm{m}$ pore size cellulose acetate filter. 
Conclusion: Our work alters the current presumption that molecular biological processes are necessary for mediating complex biological processes like cell-replication. Our observations suggest that reproduction can entirely be driven by physicochemical properties of cell constituents and laws of physical chemistry. Given the simplicity and efficiency of the process, we propose that primitive cells inhabiting early Earth (protocells) could have reproduced by this process. In our subsequent work, we were able to demonstrate from the fossil record of early Earth that this mechanism of reproduction is not merely theoretical, but protocells in-deed reproduced by these processes ${ }^{42}$.

Author contributions: DK conceived the work with input from TL, BZ, ML \& PS. DK performed laboratory experiments. AK did TEM imaging. DK \& ML performed confocal super-resolution light microscopy imaging. DK wrote the manuscript with input from TL, BZ, ML, AK \& PS. This research was funded by the European Research Council (ERC), grant agreement 616644 (POLLOX) to TL.

Acknowledgments: We would like to thank Gabriella Berthal and Markus Oster for excellent technical support. We would also like to thank Advanced Light Microscopy Facility at EMBL, Heidelberg, Ulf Schwartz from Leica Microsystems, colleagues at Ecological microbiology (Bayreuth University) and cellular biophysics laboratory (Max Planck institute for Biochemistry) for their support throughout the work. We thank Jan-Hagen Krohn for critical reading of the manuscript. 


\section{References:}

(1) Oparin, A. I. R. Inst. Chem. Rev. 1969, 2, 1-12. https://doi.org/10.1039/rr9690200001.

(2) Miller, S. L. Science. 1953, 117, 528-529.

(3) Mariani, A.; Russell, D. A.; Javelle, T.; Sutherland, J. D. A. J. Am. Chem. Soc. 2018, 140, 8657-6861. https://doi.org/10.1021/jacs.8b05189.

(4) Powner, M. W.; Gerland, B.; Sutherland, J. D. Nature 2009, 459, 239-242. https://doi.org/10.1038/nature08013.

(5) Rajamani, S.; Ichida, J. K.; Antal, T.; Treco, D. A.; Leu, K.; Nowak, M. A.; Szostak, J. W.; Chen, I. A. J. Am. Chem. Soc. 2010, 132, 5880-5885. https://doi.org/10.1021/ja100780p.

(6) Chen, I. Science. 2006, 314, 1558-1559.

(7) Zhu, T. F.; Szostak, J. W. J. Am. Chem. Soc. 2009, 131, 5705-5713.

(8) Budin, I.; Debnath, A.; Szostak, J. W. J. Am. Chem. Soc. 2012, 134. 20812-20819. https://doi.org/10.1021/ja310382d.

(9) Wu, L. J.; Lee, S.; Park, S.; Eland, L. E.; Wipat, A.; Holden, S.; Errington, J. Nat. Commun. 2020, 11, 4149. https://doi.org/10.1038/s41467-020-17988-7.

(10) Leaver, M.; Dominguez-Cuevas, P.; Coxhead, J. M.; Daniel, R. A.; Errington, J. Nature 2009, 457, 849-853.

(11) Tsai, H. H. G.; Lai, W. X.; Lin, H. Da; Lee, J. Bin; Juang, W. F.; Tseng, W. H. Biochim. Biophys. Acta-Biomembr. 2012, 1818, 2742-2755.

https://doi.org/10.1016/j.bbamem.2012.05.029.

(12) Blake, R. E.; Chang, S. J.; Lepland, A. Nature 2010, 464, 1029-1032. https://doi.org/10.1038/nature08952.

(13) Catling, D. C.; Zahnle, K. J. Sci. Adv. 2020, 6, eaax1420. 
https://doi.org/10.1126/sciadv.aax1420.

(14) Knauth, L. P. Nature 1998, 395, 554.

(15) Moore, E. K.; Jelen, B. I.; Giovannelli, D.; Raanan, H.; Falkowski, P. G. Nature

Geoscience. 2017. 10, 629-636. https://doi.org/10.1038/ngeo3006.

(16) Johnson, T. E.; Brown, M.; Kaus, B. J. P.; Vantongeren, J. A. Nat. Geosci. 2014, 7, 629-636. https://doi.org/10.1038/ngeo2019.

(17) Jülicher, F.; Lipowsky, R. Phys. Rev. Lett. 1993, 70, 2964.

(18) Mercier, R.; Kawai, Y.; Errington, J. Cell, 2013, 152, 997-1007.

(19) Narsimhan, V.; Spann, A. P.; Shaqfeh, E. S. G. J. Fluid Mech. 2015, 777, 1-26. https://doi.org/10.1017/jfm.2015.345.

(20) Kantsler, V.; Segre, E.; Steinberg, V. Phys. Rev. Lett. 2008, 101, 048101- 4. https://doi.org/10.1103/PhysRevLett.101.048101.

(21) Yanagisawa, M.; Imai, M.; Taniguchi, T. Phys. Rev. E - Stat. Nonlinear, Soft Matter Phys. 2010, 82, 051928-1-9. https://doi.org/10.1103/PhysRevE.82.051928.

(22) Yanagisawa, M.; Imai, M.; Taniguchi, T. Phys. Rev. Lett. 2008, 100, 148102-4. https://doi.org/10.1103/PhysRevLett.100.148102.

(23) Kato, A.; Tsuji, A.; Yanagisawa, M.; Saeki, D.; Juni, K.; Morimoto, Y.; Yoshikawa, K. J. Phys. Chem. Lett. 2010, 23, 3391-3395. 2010. https://doi.org/10.1021/jz101376m.

(24) Bobrovska, N.; Góźdź, W.; Kralj-Iglič, V.; Iglič, A. PLOS one. 2013, 8, 0073941. https://doi.org/10.1371/journal.pone.0073941.

(25) K. Simons, E. Ikonen, Functional rafts in cell membranes. Nature, 1997, 387, 569-572. https://doi.org/10.1038/42408.

(26) Schara, K.; Janša, V.; Šuštar, V.; Dolinar, D.; Pavlič, J. I.; Lokar, M.; Kralj-Iglič, V.; Veranič, P.; Iglič, A. Mechanisms for the Formation of Membranous Nanostructures in 
Cell-to-Cell Communication. Cellular and Molecular Biology Letters. 2009. https://doi.org/10.2478/s11658-009-0018-0.

(27) E. Sezgin, I. Levental, S. Mayor, C. Eggeling. Nat. Rev. Mol. Cell Biol. 2017, 18, 361374. https://doi.org/10.1038/nrm.2017.16.

(28) Cooper, S. Theoretical Biology and Medical Modelling. 2006, 3, 1-15. https://doi.org/10.1186/1742-4682-3-10.

(29) Mitchison, J. M. Single Cell Studies of the Cell Cycle and Some Models. Theor. Biol. Med. Model. 2005. https://doi.org/10.1186/1742-4682-2-4.

(30) Mitchison, J. M. Theor. Biol. Med. Model. 2005, 2, 1-5. https://doi.org/10.1038/nrg1615.

(31) Elowitz, M. B.; Levine, A. J.; Siggia, E. D.; Swain, P. S. Science. 2002, 297, 11831186. https://doi.org/10.1126/science.1070919.

(32) Strovas, T. J.; Sauter, L. M.; Guo, X.; Lidstrom, M. E. J. Bacteriol. 2007, 189, $7127-$ 7133. https://doi.org/10.1128/JB.00746-07.

(33) Karimi, M.; Steinkühler, J.; Roy, D.; Dasgupta, R.; Lipowsky, R.; Dimova, R. Nano Lett. 2018, 18, 7816-7821. https://doi.org/10.1021/acs.nanolett.8b03584.

(34) Schultz, Z. D.; Pazos, I. M.; McNeil-Watson, F. K.; Lewis, E. N.; Levin, I. W. J. Phys. Chem. B. 2009, 113, 9932-9941. https://doi.org/10.1021/jp9011944.

(35) Deshpande, S.; Spoelstra, W. K.; Van Doorn, M.; Kerssemakers, J.; Dekker, C. ACS Nano 2018, 12, 2560-2568. https://doi.org/10.1021/acsnano.7b08411.

(36) Hanczyc, M. M.; Fujikawa, S. M.; Szostak, J. W. Science. 2003, 302, 618-622.

(37) Zwicker, D.; Seyboldt, R.; Weber, C. A.; Hyman, A. A.; Jülicher, F. Nat. Phys. 2017, 13, 408-413. https://doi.org/10.1038/nphys3984.

(38) Vutukuri, H. R.; Hoore, M.; Abaurrea-Velasco, C.; van Buren, L.; Dutto, A.; Auth, T.; Fedosov, D. A.; Gompper, G.; Vermant, J. Nature 2020, 2020, 586, 52-56. 
https://doi.org/10.1038/s41586-020-2730-x.

(39) Levy, S.; Chevion, M. Environ. Toxicol. Chem. 2009, 28, 1380-1389.

https://doi.org/10.1897/08-518.1.

(40) Spahn, C.; Grimm, J. B.; Lavis, L. D.; Lampe, M.; Heilemann, M. Nano Lett. 2019, 19, 500-505. https://doi.org/10.1021/acs.nanolett.8b04385.

(41) Tarun, O. B.; Okur, H. I.; Rangamani, P.; Roke, S. Commun. Chem. 2020, 3, article number 17. https://doi.org/10.1038/s42004-020-0263-8.

(42) Kanaparthi, D.; Lampe, M.; Zhu, B.; Boesen, T.; Klingl, A.; Schwille, P.; Lueders, T. bioRxiv 2021, 2021.08.16.456462. https://doi.org/10.1101/2021.08.16.456462.

\section{Table of content: Lifecycle of multilamellar protocell}

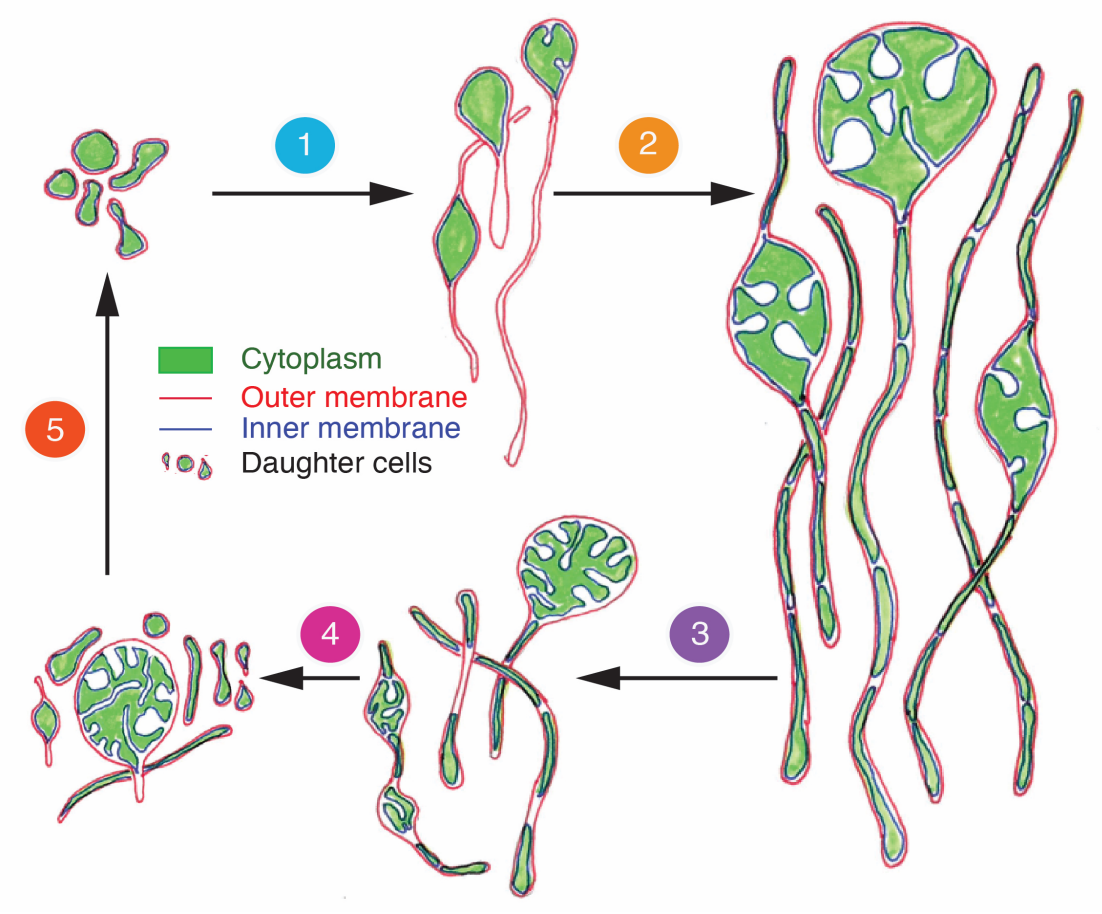

